\title{
Local and global stability of a piecewise linear oscillator
}

\author{
By M. KLeCZKA ${ }^{1}$, E. KreuzeR and W. Schiehlen ${ }^{1}$ \\ ${ }^{1}$ Institut B für Mechanik, Universität Stuttgart, Pfaffenwaldring 9,

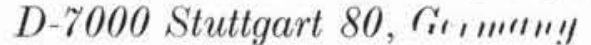 \\ ${ }^{2}$ Meerestechnik II, Universität Hamburg-Harburg, Eißendorfer Straße 42, \\ D-2100 Hamburg 90, Germany
}

\begin{abstract}
Machines and mechanisms with moving parts, subjected to periodic excitation, often show unexpected dynamic behaviour, and impacts due to their connection clearances may occur. The most simple mathematical model is a one degree-of-freedom nonlinear oscillator governed by a piecewise linear symmetric function to describe the restoring force. The system's response, which can be quite rich and complicated, is described in detail. Modern methods for a combined analytical and numerical analysis are used to study local and global bifurcation conditions, coexisting solutions and their associated domains of attraction.
\end{abstract}

\section{Introduction}

Dynamical systems theory has predominantly focused on smooth differentiable systems. By relying on such systems a considerable body of knowledge has been developed, and topological methods have given much insight into the dynamics of the overall behaviour. But discontinuous dynamical systems are common in engineering and their study has applications in a wide range of technical problems. In particular play or backlash is an omnipresent reality in machinery. Because of production tolerances, joints of mechanical systems in general are affected by play or connection clearances that may enlarge in case of wear. The resulting undesired motions often lead to $(a)$ increasing stress and strain of parts, $(b)$ reduced machine reliability and life, $(c)$ loss of precision and stability and $(d)$ undesirable noise effecting the environment.

Eliminating connection clearances by sophisticated design can, however, be very expensive and is sometimes even impossible. Increasing demands on engineering systems require, therefore, improved models and more sophisticated methods for selecting system parameters so that undesired motions could be minimised or eliminated. Such models include nonlinear and even non-differentiable elements. As the most simple prototype to analyse the dynamies of such kind of systems we consider a simple single degree-of-freedom mechanical oscillator with a piecewise linear, symmetric restoring force subjected to periodic excitation caused by an unbalanced rotor (figure 1). This can be compared with the asymmetric impact oscillator studied by Thompson \& Stewart $(1986$, ch. 14, 15) and Shaw \& Holmes $(1983 a-c)$.

The system is very simple, but it has a very complex bifurcation structure leading to irregular, chaotic motions and for certain parameter ranges it may have a variety of coexisting attracting motions (Kleczka 1991). The equations of motion include six

Phil. Trans. R. Soc. Lond. A (1992) 338, 533-546 

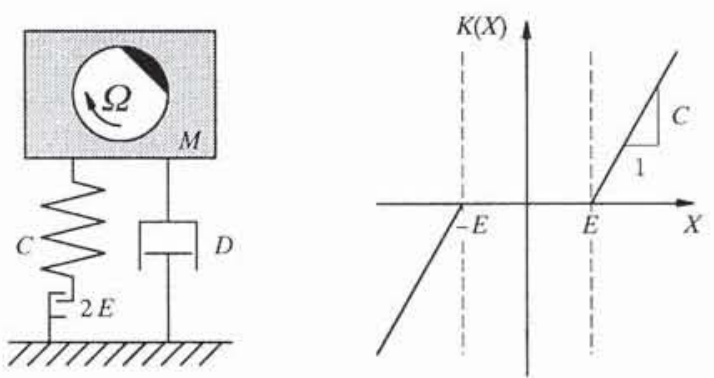

Figure 1. Mechanical model and piecewise linear symmetric spring characteristic.

physical parameters: mass $M$, play $E$, coefficients of spring $C$ and damper $D$, amplitude $A$ and frequency $\Omega$ of excitation. Normalization leads to the nondimensionalized equations of motion

$$
\ddot{x}+2 d \dot{x}+k(x)=a \cos t,
$$

containing only three generalized parameters of spring, damper and amplitude of excitation

$$
c:=C / M \Omega^{2}, \quad d:=\frac{1}{2} D / M \Omega, \quad a:=A / E M \Omega^{2} .
$$

The normalized piecewise linear function of restoring force is

$$
k(x):= \begin{cases}c(x-1) & \text { if } x \geqslant 1, \\ 0 & \text { if }-1<x<1, \\ c(x+1) & \text { if } x \leqslant 1 .\end{cases}
$$

\section{Extended state space}

Methods for the analysis of nonlinear dynamic systems usually require a representation of the system as a set of autonomous, first-order ordinary differential equations. To apply these methods, the given single, second-order non-autonomous system (1) has to be transformed appropriately. To preserve the property of noncrossing trajectories the system has to be rewritten as an autonomous system by introducing time as an additional state variable (figure $2 a$ ). In the case of a periodic time dependence, a common way to transfer (1) to the desired form is to establish an extended, cylindrical state space,

$$
x=(x, y=\dot{x}, \theta=t \bmod 2 \pi) \in R^{2} \times S^{1}, \quad S^{1}=[0,2 \pi),
$$

where $x$ represents the position, $y$ gives the velocity and $\theta$ stands for the circular coordinate and is to be interpreted as the phase angle of excitation. The resulting set of first order autonomous differential equations is given by

$$
\left[\begin{array}{c}
\dot{x} \\
\dot{y} \\
\dot{\theta}
\end{array}\right]=\left[\begin{array}{c}
y \\
-2 d y-k(x)+a \cos \theta \\
1
\end{array}\right] .
$$

The subspaces, where the piecewise linear function is not smooth,

$$
\begin{aligned}
& S^{+1}=\left\{(x, y, \theta) \in R^{2} \times S \mid x=+1.0\right\}, \\
& S^{-1}=\left\{(x, y, \theta) \in R^{2} \times S \mid x=-1.0\right\},
\end{aligned}
$$

are called switching surfaces. Intersecting one of these surfaces, the system's dynamies changes non-smoothly from one linear behaviour to another. Although the 
system is almost everywhere linear, the global behaviour is, of course, nonlinear. Figure 2 shows two different representations of the extended state space including the switching surfaces.

There exist no global analytical solutions in closed form and therefore the system has to be integrated numerically. Instead of studying the continuous time system or flow one can obtain considerably more insight into the dynamics by analysing an associated discrete time system or map. This is especially true for the analysis of orbital stability of periodic solutions.

\section{Poincaré map}

An essential simplification and reduction of the analysis of (5) can be achieved by discretization of the time-continuous description by means of a Poincaré surface of section. One major problem is to set up a proper Poincaré surface of section in state space. There are two natural ways to discretize the flow of the set of ordinary differential equations for the system under consideration: (i) the stroboscopic map which is obtained by measuring the position and the velocity at a fixed value of the phase angle $\theta$; (ii) one of the switching surfaces which maps the phase angle and the velocity at an intersection onto the phase angle and the velocity of the next intersection. For periodically excited systems it is common to establish a Poincaré surface of section $\Gamma$ at some arbitrary but fixed phase angle $\theta_{0}$ :

$$
\Gamma=\left\{(x, y, \theta) \mid \theta=\theta_{0}\right\} .
$$

In the following the surface of section is located at $\theta_{0}=0$. Each trajectory crosses $\Gamma$ transversally once per period of excitation. The main advantage of the stroboscopic map or Poincaré surface of section $\Gamma$ is that it is globally transversal to the flow of (5).

On the other hand, if a system is non-smooth on some surface in state space, it may be appropriate to watch the system's state crossing this surface, i.e. to use one of the switching surfaces as Poincaré surface of section. In the given case, switching surfaces are not globally transversal to the flow : for $y>0$ trajectories go to the right, for $y<0$ trajectories go to the left and for $y=0$ trajectories touch the switching surfaces tangentially, i.e. non-transversally. As Poincaré surfaces of section have to be transversal, only parts of switching surfaces are suitable. One set of possible Poincaré surfaces of section is:

$$
\left.\begin{array}{l}
\Sigma^{1}=\{(x, y, \theta) \mid x=+1.0, y<0\}(=\Sigma), \\
\Sigma^{2}=\{(x, y, \theta) \mid x=-1.0, y<0\}, \\
\Sigma^{3}=\{(x, y, \theta) \mid x=-1.0, y>0\} . \\
\Sigma^{4}=\{(x, y, \theta) \mid x=+1.0, y>0\} .
\end{array}\right\}
$$

Because the system is perfectly symmetric, all of the four Poincaré surfaces of section $\Sigma^{1 \ldots 4}$ are equivalent. For that reason only $\Sigma \equiv \Sigma^{1}$ needs to be treated without loss of generality.

In practice, switching points have to be determined by an iterative procedure. These points can be used as Poincaré points without further computational expense. This is a minor advantage compared to the Poincaré surface of section $\Gamma$, where the system's state has to be determined passing the phase angle $\theta_{0}$. The main disadvantage of the Poincaré surface of section $\Sigma$ is the non-global transversality. It leads to discontinuities of the respective Poincaré map and consequently to severe

Phil. Trans. R. Soc. Lond. A (1992) 


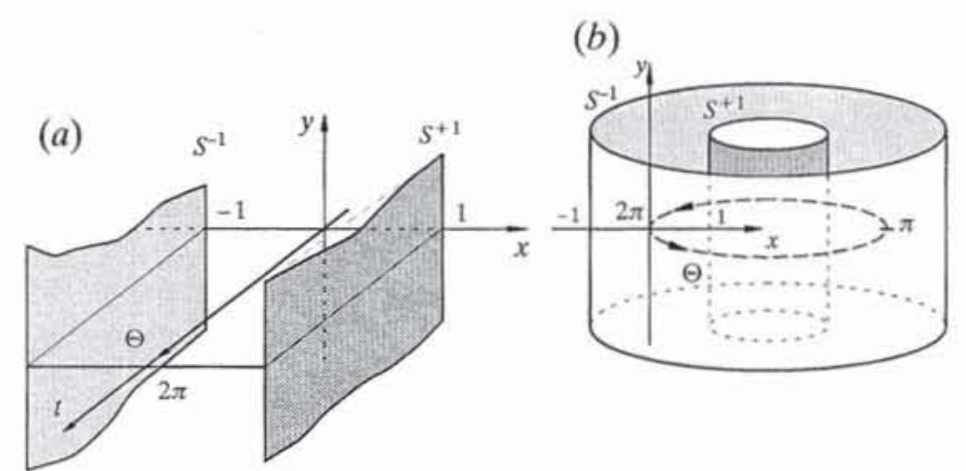

Figure 2. (a) Linearly and $(b)$ cylindrically extended state space.
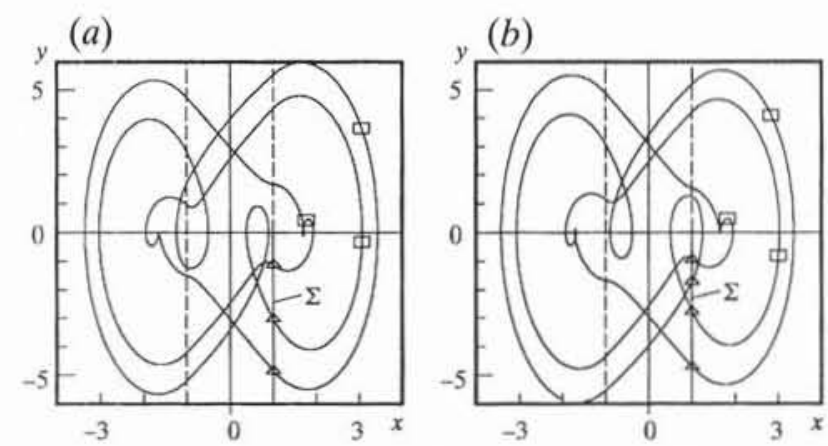

Figure 3. Coexisting, pairwise point-symmetric solutions. $\Gamma$-Poinearé points, $\square: \Sigma$-Poincaré points, $\Delta$ : for $c=12.0, d=0.15, a=10$.

numerical and theoretical problems. This remarkable fact has not received very much attention in the literature, although often non-global Poincaré surfaces of section are used disregarding the related problems. Effects of discontinuities due to non-global Poincaré surfaces of section may be $(a)$ problems in interpreting the period of an orbit, $(b)$ problems in calculating invariant manifolds and $(c)$ anomalous bifurcations, e.g. fictitious bifurcation from a period three (P3) solution to a solution of period four (P4).

The first difficulty can be demonstrated easily by a simple example. For the set of parameter values $(c=12.0, d=0.15, a=10.0)$ two solutions of period $3 \times 2 \pi$ coexist (figure 3).

In the globally transversal Poincaré section $\Gamma$ these solutions are represented as a pair of P3 solutions. This is not the case for the non-global Poincaré section $\Sigma$. The left solution (figure $3 a$ ) will be represented as a P3 solution whereas the corresponding right solution (figure $3 b$ ) comes up as a P4 solution. Although both solutions are point-symmetric to each other with respect to the origin and of period $3 \times 2 \pi$ in the continuous case, discretization by means of a Poincaré section $\Sigma$ that is not globally transverse, leads to different periodicity and loss of symmetry properties. The discontinuity of the adjoint Poincaré map leading to this problem is made apparent by simulation in the neighbourhood of the discontinuity. Figure 4 shows trajectories starting in the Poincaré surface of section $\Sigma$ with fixed $y$-coordinate and slightly varying phase angle $\theta$.

Figure $4 a$ gives an overview on the situation close to the discontinuity. Figure $4 b$ is a detail of a simulation immediately before the jump. The loop does not touch the Poincaré section. The next simulation, figure $4 c$, reflects the critical situation. The loop seems to touch the Poincaré section and two image points are possible, depending on whether the loop crosses the Poincaré section or not. Finally, further 

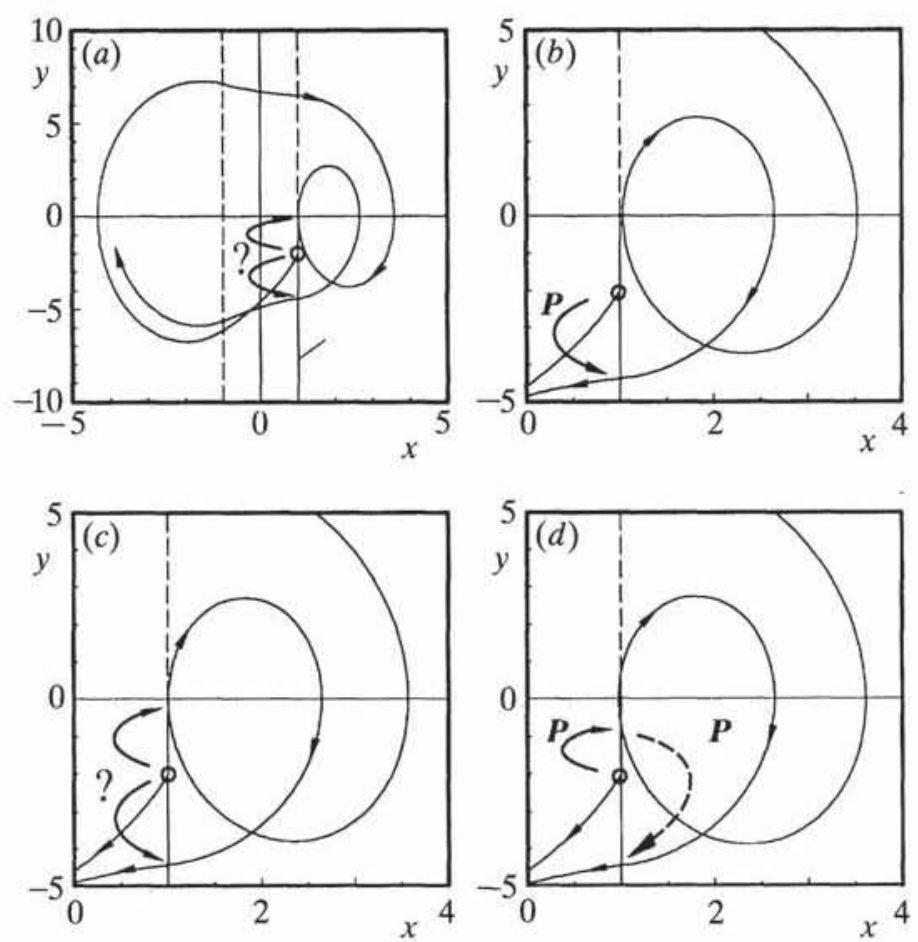

Figure 4. Trajectories in the neighbourhood of a discontinuity of the Poincaré map $P_{\Sigma} .(a)$ Complete trajectory, $(b)$ immediately before the discontinuity, $(c)$ at the discontinuity, $(d)$ immediately after the discontinuity.

variation of the phase angle makes the situation clear: the loop crosses the Poincaré section producing a 'new' image point, without continuous connection to the old one. The discontinuity occurs in general in the case of a Poincaré section, that is not globally transverse.

\section{Global analysis}

The global analysis of nonlinear dynamical systems in general requires large-scale numerical investigations. Global stability analysis with respect to initial conditions leads to the problem of the determination of long-term behaviour, i.e. attractors, and their domains of attraction.

The most efficient approach to such analysis involves discretization of state and time, the so-called cell mapping method (Hsu 1981; Kreuzer 1987). Analysis of the coexistence situation (figure 3) reveals an intertwining of the respective domains of attraction, which is an indication that the system is sensitive with respect to small changes of initial conditions in the sense that arbitrarily close starting points eventually produce large changes in the long-term behaviour (figure 5).

Individual trajectories are therefore not reproducible in the presence of physical or numerical noise and the emphasis is placed, rather, on the overall features of ensembles of trajectories. Fixing an initial condition in the finely intertwined régime to some limited precision may lead to unpredictable long-term behaviour; it is impossible to determine which of the two possible P3 solutions will be approached in the long run. Sensitive dependence on initial conditions is a typical property of chaotic dynamics.

A global analysis in parameter space can be carried out with a calculation of Lyapunov exponents and bifurcation diagrams. Lines in bifurcation diagrams represent periodic solutions changing under variation of the control parameter 


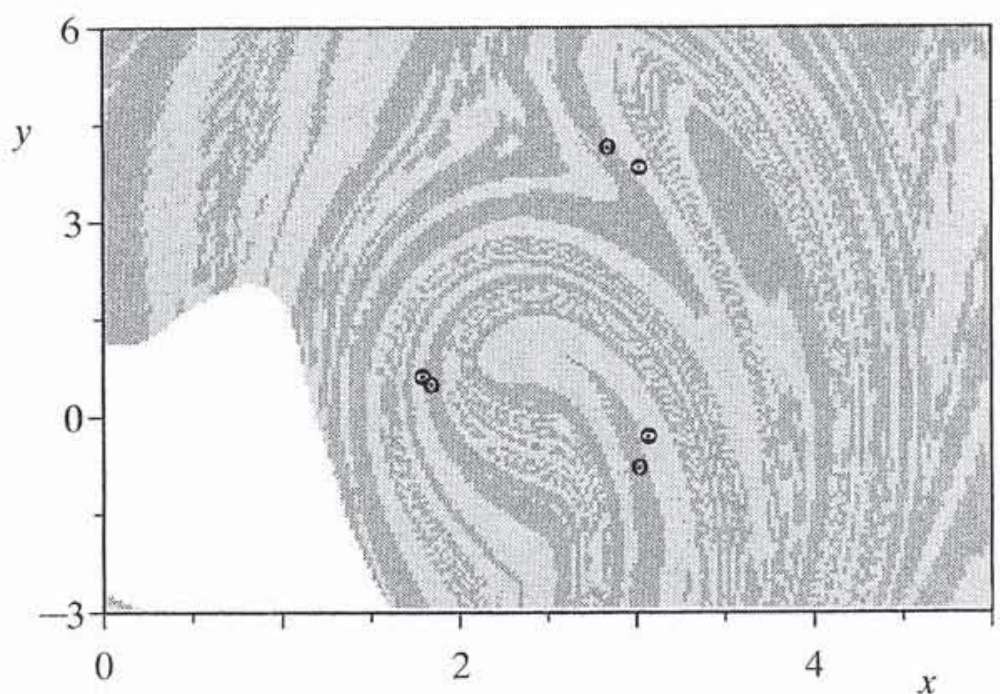

Figure 5. Coexisting P3 solutions and intertwining régime in the domains of attraction.
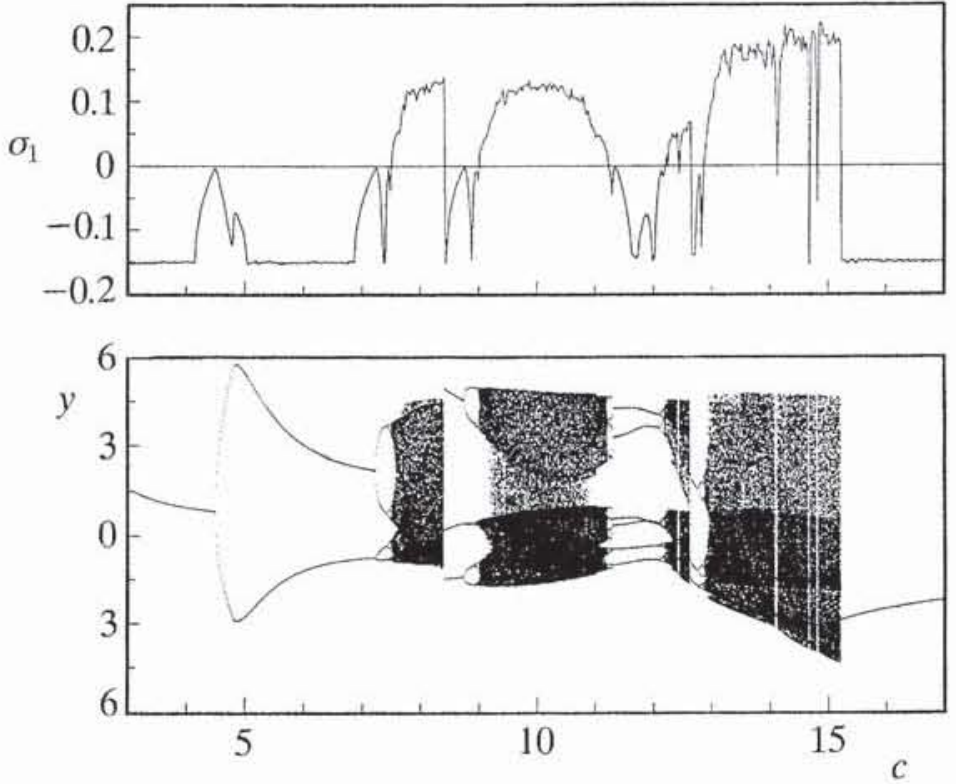

Figure 6. Bifurcation diagram and Lyapunov exponent $\sigma_{1}$ for variation of the stiffness ratio $c$.

steadily or, in case of bifurcation, unsteadily. The corresponding Lyapunov exponent is negative, indicating stable, convergent long-term behaviour. Chaotic dynamics produces non-periodic sequences of points in the bifurcation diagram leading to wide, grey-patterned structures. The corresponding largest Lyapunov exponent is positive, indicating divergent, unpredictable dynamics. The analysis for variation of the stiffness ratio $c$ is shown in figure 6 .

In parameter space, the P3 solution (Yorke \& Li 1975) is just a periodic window within the chaotic régime.

\subsection{Homoclinic structures and horseshoe dynamics}

Chaotic dynamics is a phenomenon of systems that obey deterministic laws, but whose behaviour is unpredictable. But how can this happen? Determinism means: identical initial conditions lead to identical results. In the classical sense determinism is equivalent to predictability. But in the classical sense determinism has been considered only in the strong sense, meaning nearly identical initial conditions lead 
emergence of homoclinic structure
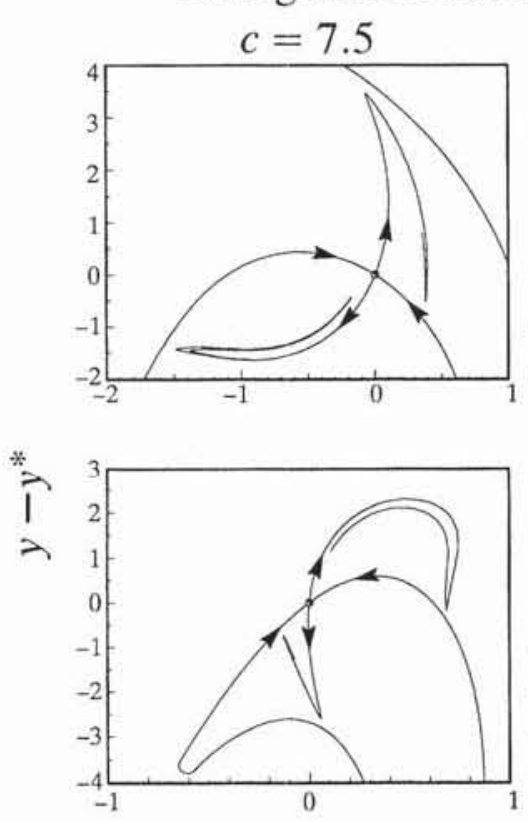

chaotic attractor
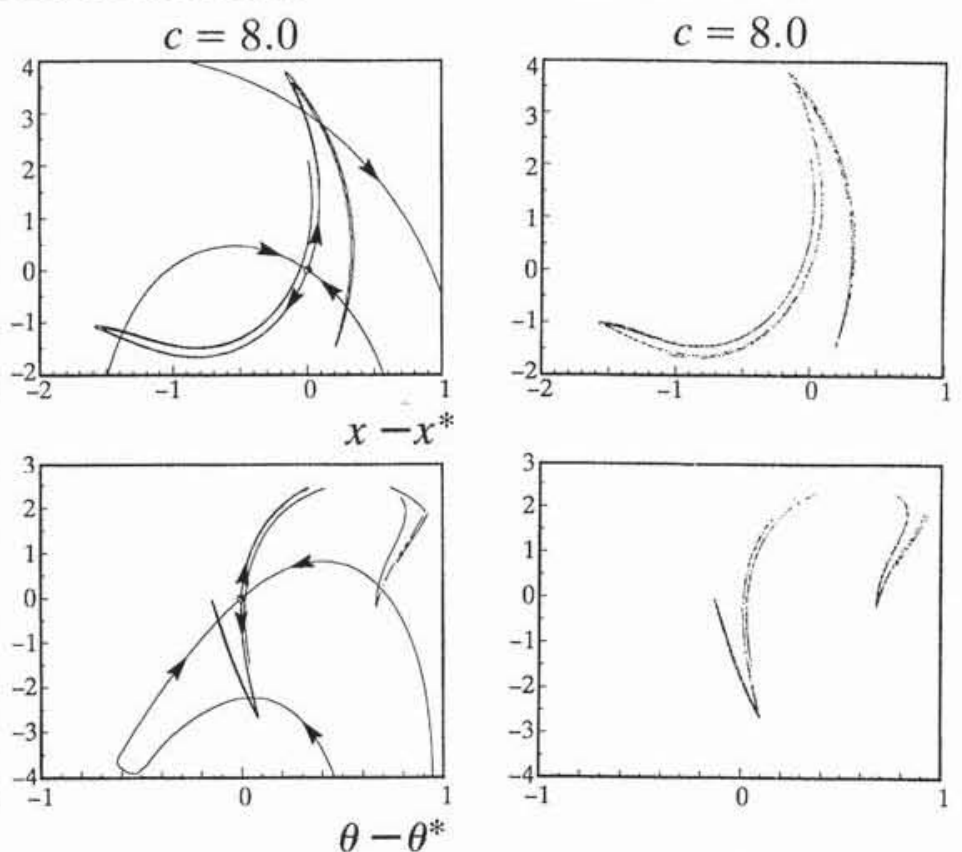

Figure 7. Emergence of homoclinic points and the respective chaotic attractor.

to nearly identical results. Chaotic dynamics is deterministic, but not strongly deterministic. Chaotic systems react sensitively to each variation of initial conditions. In the real world initial conditions are never precisely determined and can only be fixed within a certain level of uncertainty. Inherent divergence of chaotic systems produces, in time, diverging uncertainty up to a point where the information about the state of the system, given at some initial time, has become worthless: knowing the initial conditions, after some time, the actual state has become unpredictable. The most illustrative prototype of such behaviour is the Smale horseshoe map. It can be shown that Poincaré maps generating homoclinic structures are locally topologically equivalent to the Smale horseshoe map. Homoclinic structures emerge when stable and unstable invariant manifolds of fixed points of saddle type intersect transversally. In this case, the map under consideration has locally all prototypical chaotic properties of the horseshoe map: $(a)$ existence of a countable infinite number of periodic orbits of saddle type; $(b)$ existence of an uncountable infinite number of non-periodic orbits; $(c)$ existence of a dense orbit; and $(d)$ sensitive dependence on initial conditions.

For the backlash oscillator under consideration an unstable, saddle type, symmetric periodic solution plays the decisive role; its stable and unstable invariant manifolds intersect, leading to homoclinic structures with significant influence on the dynamics of the system (figure 7 ).

Homoclinic structures and horseshoes generate chaotic behaviour, but they do not guarantee asymptotic, long-term chaotic motions. The invariant set of the horseshoe map is not attractive; in contrast it is unstable. In general it is not possible to prove the existence of a chaotic attractor. In the given case (figure 7) a chaotic attractor seems to correspond to the unstable invariant manifold containing the chaotic horseshoe set. Furthermore, in this case the emergence of homoclinic points coincides with the emergence of a chaotic attractor. As already mentioned this is not true in general. As the global topological structure of the system changes qualitatively, the system undergoes a global bifurcation.

Phil. Trans. R. Soc. Lond. A (1992) 


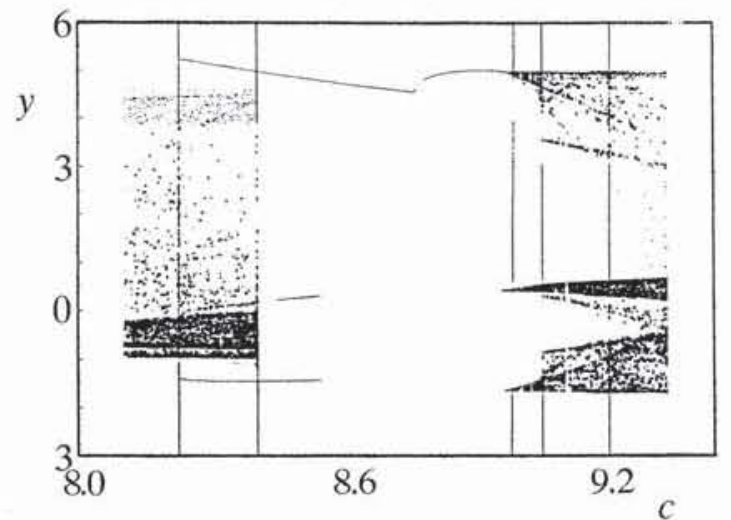

Figure 8. Magnification of the bifurcation diagram of figure 6 in the P3 range.
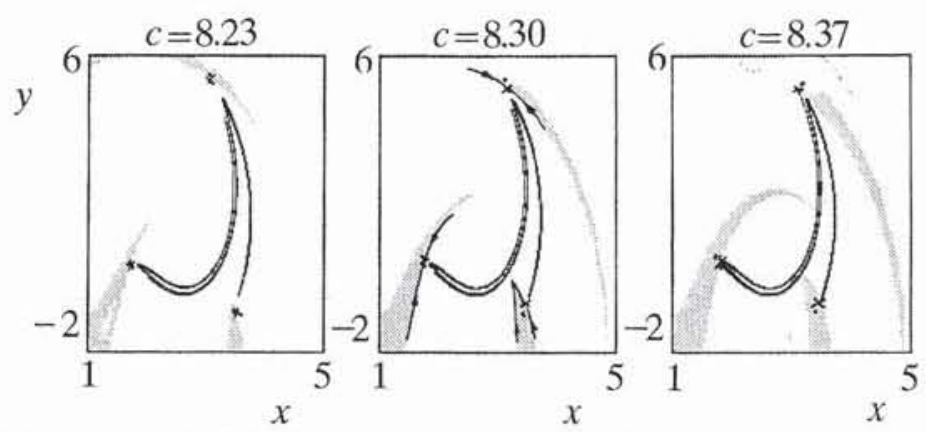

Figure 9. Backlash oscillator approaching an exterior crisis.

Global bifurcations are not smooth. Consequently, the resulting changes of the dynamies are not smooth, and often the system jumps, collapses or explodes: in other words its behaviour changes drastically. The different terms used to describe such behaviour reflect this: catastrophe, explosion, crisis, etc. We now focus on crisis phenomena, leading to sudden unexpected changes of the chaotic dynamics.

\subsection{Crisis phenomena}

Looking at the bifurcation diagram (figure 6 ) one realizes that there are sudden disappearances and sudden blow-ups of the chaotic attractor. A magnification of the P3 window (figure 8) makes this behaviour clearly visible.

Following Grebogi et al. (1983), one distinguishes two different classes of crisis phenomena: $(a)$ interior crises, producing blow-ups within the chaotic range; $(b)$ exterior crises, leading to sudden disappearances of chaotic attractors.

An exterior crisis occurs at a parameter value $c \approx 8.4$. The chaotic attractor vanishes, because it collides with an unstable periodic solution. Simultaneously, the separatrix, dividing the domains of attraction of the coexisting chaotic and the P3 solutions, touches the chaotic attractor and induces loss regions within the former attractor leading to a global loss of attractivity of the chaotic set. Figure 9 shows how basin boundary and unstable periodic points approach the chaotic attractor and finally destroy it by an exterior crisis.

An interior crisis can be observed at a parameter value of $c \approx 9.17$. At this point the three piece chaotic attractor suddenly blows up resulting in a one piece chaotic attractor. This is not only an enlargement of the chaotic dynamies, but also a qualitative change: the interior crisis marks the end of the realm of period three. The final stage of chaotically disturbed P3 behaviour, reflected by a three piece chaotic attractor is definitely terminated (figure 10). 

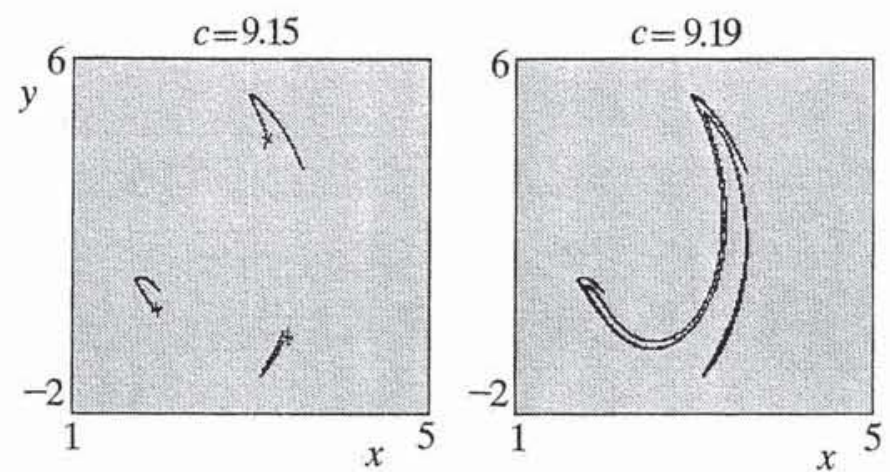

Figure 10. Cell mapping results of an interior erisis.

It is an interesting result that both the establishment and termination of the period three intermezzo is triggered by the same unstable P3 solution (Kleczka et al. $1990 a)$.

\section{Local stability analysis}

The basic foundation of the local stability analysis of nonlinear dynamical systems with respect to a vector of control parameters $\boldsymbol{\mu}$ is the classical bifurcation theory. The bifurcation theory applies to continuous systems given as a set of ordinary, autonomous differential equations

$$
\dot{x}=f(x, \mu)
$$

and to discrete systems given as a map

$$
x_{k+1}=P\left(x_{k}, \mu\right) .
$$

The prescribed steps of the analysis include (Troger \& Steindl 1991) the following.

1. Determination of the bifurcation parameter value $\boldsymbol{\mu}^{*}$, where the stability of the solution is critical (i.e. not determined by linear analysis).

2. Reduction to the bifurcation system by elimination of non-critical state variables.

3. Transformation to normal form and identification of resonance terms governing the system's dynamics.

4. Sensitivity analysis with respect to imperfections through a universal unfolding.

5 . Investigation of robustness with respect to large scale parameter variations.

In the continuous case, stability properties of stationary solutions $\dot{x}=\mathbf{0}$ can be determined. The same procedure can not be applied directly to periodic solutions of differential equations. In terms of a Poincaré map, the problem of studying the stability of a periodic solution is reduced to the problem of stability of a fixed point of the map as was mentioned above. But bifurcation analysis of maps relies on explicitly given mapping equations including symbolic control parameters. Unfortunately, there exist no general methods applicable to arbitrary differential equations for constructing the associated Poincaré map analytically. It will be shown how to overcome this difficulty by using a computer aided symbolic-numeric procedure generating a parametrized power series approximation of the Poincaré map.

The classical bifurcation analysis as outlined above can be built upon this approximation. The Taylor series approximation turns out to be a stable foundation, allowing a general bifurcation analysis of oscillatory engineering systems, such as the backlash oscillator. 


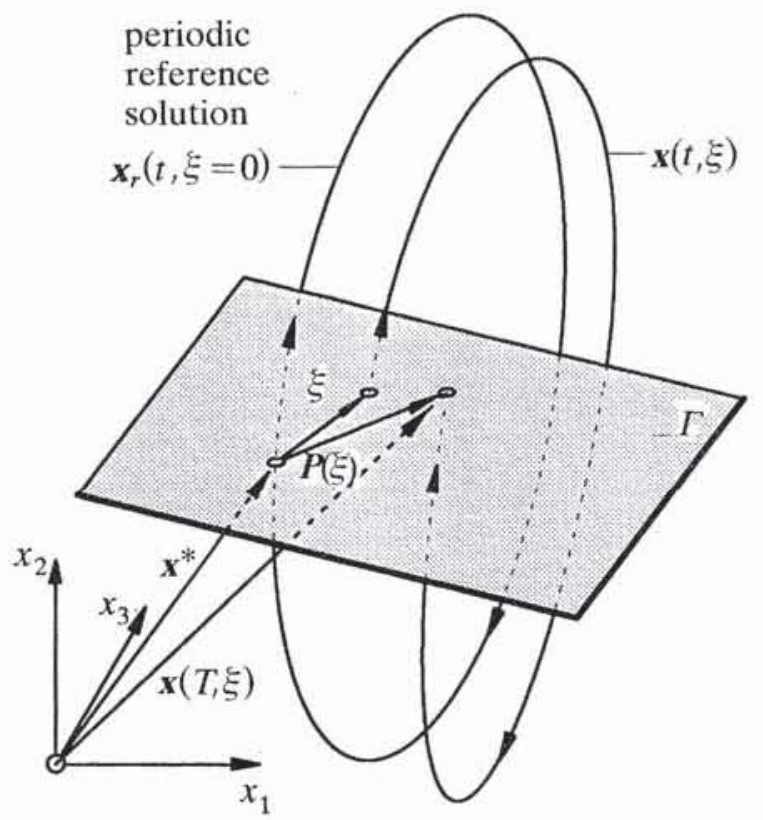

Figure 11. Poincaré map: periodic reference solution and its neighbourhood.

\subsection{Local Taylor approximation of the Poincaré map}

The Poincaré map can be determined only by numerical integration of the underlying continuous system. A local approximation of the Poincaré map in the neighbourhood of a fixed point can be developed starting from a periodic reference solution, figure 11 .

If initial conditions in the Poincaré section are described by a vector $\xi$, the reference solution of period $T$ fulfils

$$
x(t=0, \xi=0)=x(T, 0)=x^{*},
$$

and for the respective Poincaré map we have

$$
P(\xi=0)=0 .
$$

A local approximation of the Poincaré map, $G(\xi)$, can be given as Taylor series expansion

$$
G(\xi)=P^{\prime} \xi+\frac{1}{2} P^{\prime \prime}\{\xi, \xi\}+\frac{1}{6} P^{\prime \prime \prime}\{\xi, \xi, \xi\} \ldots+1 / k ! P^{(k)}\{\xi, \ldots, \xi\},
$$

where $\{\boldsymbol{\xi}, \ldots, \boldsymbol{\xi}\}$ denotes the outer product. If $\boldsymbol{x}(t, \boldsymbol{\xi})$ describes the solution of

$$
\dot{x}=f(x), \quad x(t=0)=x^{*}+\xi,
$$

the Poincaré map is defined by

$$
P(\xi)=x(T, \xi)-x^{*} .
$$

The unknown jacobian $P^{\prime}$ can be expressed as

$$
P^{\prime}=P^{\prime}(T, 0)=\left.\frac{\partial x(T, \xi)}{\partial \xi}\right|_{\xi=0}=\left.\frac{\partial x(t, \xi)}{\partial \xi}\right|_{\substack{t=T \\ \xi=0}} .
$$

The connection to the system's differential equation

$$
\dot{x}=f(x)
$$

Phil. Trans. R. Soc, Lond. A (1992) 
Table 1. Number of differential equations

\begin{tabular}{rcrrrrrr}
\hline$p$ & $n=2$ & $n=3$ & $n=4$ & $n=5$ & $n=6$ & $n=7$ & $n=8$ \\
\hline 2 & 14 & 39 & 84 & 155 & 258 & 399 & 584 \\
3 & 30 & 120 & 340 & 780 & 1554 & 2800 & 4650 \\
4 & 62 & 363 & 1364 & 3905 & 9330 & 19607 & 37448 \\
\hline
\end{tabular}

is made by differentiation with respect to $\xi$ :

$$
\frac{\mathrm{d}}{\mathrm{d} t}\left(\frac{\partial \boldsymbol{x}(t, \boldsymbol{\xi})}{\partial \xi}\right)=\frac{\partial f(\boldsymbol{x}(t, \boldsymbol{\xi}))}{\partial \xi}=\frac{\partial f(x)}{\partial \boldsymbol{x}}\left(\frac{\partial \boldsymbol{x}(t, \boldsymbol{\xi})}{\partial \xi}\right) .
$$

For an $n$-dimensional dynamical system this is a system of $n \times n$ first-order differential equations. With the initial conditions

$$
P^{\prime}(t=0, \xi=0)=\frac{\partial \boldsymbol{x}(t=0, \xi=0)}{\partial \xi}=\left[\begin{array}{lll}
1 & & 0 \\
& \ddots & \\
0 & & 1
\end{array}\right],
$$

numerical integration up to $t=T$ leads to

$$
\boldsymbol{P}^{\prime}=\boldsymbol{P}^{\prime}(t=T, \boldsymbol{\xi}=\mathbf{0})=(\partial \boldsymbol{x} / \partial \boldsymbol{\xi})(t=T, \boldsymbol{\xi}) .
$$

Further derivatives of the system's differential equation (17) supply ordinary differential equations for $\boldsymbol{P}^{\prime \prime}, \boldsymbol{P}^{\prime \prime \prime}, \ldots$.

A more compact formulation to derive the equations for the sought-after terms is given in index notation. If we define

first-order terms are

$$
\bullet{ }_{, i}=\partial \bullet / \partial \xi_{i}, \quad \bullet{ }_{i}=\partial \bullet / \partial x_{i},
$$

$$
\dot{x}_{i, j}=f_{i, j} .
$$

Application of the chain rule yields

$$
\dot{x}_{i, j}=f_{i, a} x_{a, j} .
$$

For second-order terms, equation (22) has to be differentiated again with respect to $\xi_{i}$,

$$
\begin{aligned}
\dot{x}_{i, j \hat{k}} & =\left(f_{i, a} x_{a, j}\right)_{\hat{k}}=f_{i, a \hat{k}} x_{a, j}+f_{i, a} x_{a, j \hat{k}} \\
& =f_{i, a b} x_{b, \hat{k}} x_{a, \hat{j}}+f_{i, a} x_{a, \hat{k} \hat{k} .}
\end{aligned}
$$

The next steps can be done equivalently. For third-order terms, the result is

$$
\begin{aligned}
\dot{x}_{i, \hat{j} \hat{k} \hat{\imath}}= & f_{i, a b c} x_{c, \hat{\imath}} x_{b, \hat{k}} x_{a, \hat{\jmath}}+f_{i, a b} x_{b, \hat{k} \hat{\imath}} x_{a, \hat{j}}+f_{i, a b} x_{b, \hat{k}} x_{a, \hat{\imath} \hat{l}} \\
& +f_{i, a b} x_{b, \hat{\imath}} x_{a, \hat{\jmath} \hat{k}}+f_{i, a} x_{a, \hat{\jmath} \hat{k} \hat{\imath} .}
\end{aligned}
$$

The only non-vanishing initial conditions are

$$
x_{i, j}(t=0)=\delta_{i j} .
$$

An important point is that this systematic index formulation can be fully automated using computer algebra. A MAPLE-program (Haack 1990) generates the complete set of differential equations (17), (22), (23), (24) up to an approximation order of $p=3$ for systems of arbitrary dimension $n$. Table 1 gives the correlation between approximation order $p$, system's dimension $n$ and the number of coupled differential equations to be derived and solved.

Phil. Trans. R. Soc. Lond. A (1992) 
An approximation of the Poincaré map is not very useful if the influence of parameters cannot be studied. Therefore, parameters have to be included in a symbolic way. This is possible by a simple extension of the state space. The control parameters $\mu_{i}$ under variation can be interpreted as state variables

$$
\dot{x}=f(x, \mu), \quad \dot{\mu}=0 .
$$

Now bifurcations with respect to parameter fluctuations can be studied generating a local Taylor series approximation of the Poincaré map of the extended system (26). More details are given in Kleczka et al. $(1990 \mathrm{~b})$.

It is possible to apply the outlined procedure to the given piecewise linear oscillator without considering the special structure of the restoring force (3). But the result is wrong. Only the linear part of the local approximation is correct. Higher order terms with respect to state variables vanish, as the system behaves like a linear, time-variant system. The piecewise linear system changes at switching points, defined by the periodic reference trajectory. The nonlinearity of the system consists of the state dependence of the switching points. The variation of switching points with respect to variations of the solution are not taken into account. The only way to cover the nonlinear behaviour within the presented scheme is to approximate the piecewise linear system by a closed form nonlinear system. Defining a function

$$
h\left(x ; x_{0}, c_{s}\right)=\frac{1}{2}+1 / \pi \arctan \left[c_{s}\left(x-x_{0}\right)\right],
$$

the piecewise linear system (4) can be approximated by

$$
\left[\begin{array}{c}
\dot{x} \\
\dot{y} \\
\dot{c} \\
\dot{d}
\end{array}\right]=\left[\begin{array}{c}
y \\
-2 d y-c\left(x_{1}-1\right)\left(\frac{1}{2}+1 / \pi \arctan \left[c_{8}\left(x_{1}-1\right)\right]\right) \\
-c\left(x_{1}+1\right)\left(\frac{1}{2}+1 / \pi \arctan \left[c_{s}\left(-x_{1}-1\right)\right]\right)+a \cos t \\
0 \\
0
\end{array}\right] .
$$

Driving $c_{s} \rightarrow \infty$, the closed form nonlinear system asymptotically approaches the piecewise linear system and the coefficients describing the approximation asymptotically approach the coefficients of the correct, nonlinear approximation of the piecewise linear system.

\subsection{Bifurcation analysis}

Having gained a proper nonlinear approximation of the piecewise linear system, the bifurcation analysis is standard. In particular for such a simple, low-dimensional system only a few types of bifurcations are possible: bifurcations, where one real eigenvalue of the linearized system crosses the unit circle. Hopf bifurcations and higher order degenerate cases are impossible and normal form transformation is not necessary. After the critical parameter has been determined by an iterative procedure, the critical system has to be reduced to the bifurcation system, which is in this special case a scalar difference equation, describing the dynamics on the centre manifold. This equation encloses all relevant information about the type of stability in the critical case.

The method used to reduce the system is the centre manifold formalism. The centre manifold formalism relies on power series expansions of the given equation and generates a power series expansion of the bifurcation system. It is important to 
note that the procedure can be automated completely; again taking advantage of computer algebra systems (Rand \& Armbruster 1987; Kleczka 1989). For the analysis of the pitchfork bifurcation $(c \approx 4.485)$ the procedure delivers an equation including terms up to order three

$$
x_{k+1}=x_{k}-0.02 x_{k}^{3} .
$$

The coefficient of the linear term is equal to plus one, the coefficient of the quadratic term vanishes and the coefficient of the cubic term is negative; the critical system is stable and equation (29) represents a stable pitchfork bifurcation. Beyond the bifurcation point the previous stable point symmetric P1 solution becomes unstable and two stable pairwise point symmetric P1 solutions emerge.

Further increase of the spring parameter value leads to a period doubling flip bifurcation. The corresponding bifurcation system for $(c \approx 7.23)$ is

$$
x_{k+1}=-x_{k}-0.2455 x_{k}^{2}-0.0168 x_{k}^{3},
$$

where the coefficient $a_{1}=-1$ indicates alternating behaviour that is typical for flip bifurcations. The original solution becomes unstable and no other stable P1 solution appears. Instead, a solution of double period is generated. The stability of the double mapping

$$
x_{k+2}=x_{k}-0.087 x_{k}^{3}
$$

is guaranteed by the negative coefficient of the cubic term. Consequently, the single mapping (30) undergoes a supercritical flip bifurcation producing a stable solution of double periodicity.

\section{Conclusions}

A periodically forced one degree-of-freedom oscillator with piecewise linear symmetric restoring force has been studied. In contrast to the analysis of linear systems the analysis of nonlinear systems comprises local and global properties. Whereas a simple local stability analysis, i.e. solution of a linear eigenvalue problem, suffices to determine the global behaviour of linear systems, nonlinear systems require a careful local analysis plus additional global investigations. This task is significantly more difficult than a linear analysis. The related problems are by no means solved. To study oscillatory behaviour and bifurcation structures the continuous system has been discretized by Poincaré maps. The interpretation of periodic orbits of the map depends on the choice of the surface of section. The global analysis requires in general large scale numerical simulations, e.g. the determination of asymptotic solutions and their domains of attraction and the verification of homoclinic structures. The local stability analysis is based upon an approximation of the Poincaré map including system parameters in a symbolie way. A way to master local and global stability analysis in general is visible. The way is a computer aided numeric-symbolic approach. High-performance numerical computers (such as vector and parallel processors on the one side and analytical machines carrying out symbolic formalisms by means of computer algebra on the other) provide powerful basic methods to overcome problems of nonlinear dynamics in a systematic, straightforward manner. The fundamental steps have been outlined in this paper for a simple example. In principle, this procedure is applicable to more complex engineering systems; including, for example, those found in gear boxes (Pfeiffer 1992; Weidner \& Lechner 1990).

Phil. Trans. R. Soc. Lond. A (1992) 


\section{References}

Grebogi, C., Ott, E. \& Yorke, J. 1983 Crises, sudden changes in chaotic attractors, and transient chaos. Physica D7, 181-200.

Haack, C. 1990 Rechnergestützte Verfahren zur Analyse nichtlinearer dynamischer Probleme. Diplomarbeit DIPL-28, Universität Stuttgart.

Hsu, C. S. 1981 A Generalized theory of cell-to-cell mapping for nonlinear dynamical systems. J. appl. Mech. 48, 634-642.

Kleczka, M. 1991 Methoden zur Verzweigungsanalyse mit Anwendung auf einen Spielschwinger. Fortschr.-Ber. VDI Reihe 11 no. 153. Düsseldorf: VDI-Verlag.

Kleczka, M., Kreuzer, E. \& Wilmers, Ch. $1990 a$ Crises in mechanical systems. In Proc. IUTAM Symp. on Nonlinear Dynamics in Engineering Systems W. Schiehlen, pp. 141-148. Berlin: Springer.

Kleczka, M., Kleczka, W. \& Kreuzer, E. $1990 b$ Bifurcation analysis: a combined numerical and analytical approach. In Proc. NATO Adv. Res. Workshop on Continuation and Bifurcations: Numerical Technique and Applications D. Roose, B. de Dier, A. Spence, pp. 123-137. Dordrecht: Kluwer Academic Publishers, pp. 123-137.

Kleczka, W. 1989 Einsatz von Computer-Algebra zur Analyse nichtlinearer dynamischer Probleme. Studienarbeit STUD-50, Universität Stuttgart.

Kreuzer, E. 1987 Numerische Untersuchung nichtlinearer dynamischer Systeme. Berlin: Springer.

Pfeiffer, F. 1992 Dynamical systems with time-varying or unsteady structure, Z. angew. Math. Mech. 71. (In the press.)

Rand, R. H. \& Armbruster, D. 1987 Perturbation methods, bifurcation theory and computer algebra. New York: Springer.

Shaw, S. W. \& Holmes, P. J. 1983 a A periodically forced impact oscillator with large dissipation. ASME J. appl. Mech. 50, 849-857.

Shaw, S. W. \& Holmes, P. $1983 b$ A periodically forced piecewise linear oscillator. J. Sound Vib. 90 , $129-144$.

Shaw, S. W. \& Holmes, P. $1983 c$ Periodically forced linear oscillator with impacts: chaos and longperiod motions. Phys. Rev. Lett. 51, 623-626.

Thompson, J. M. T. \& Stewart, H. B. 1986 Nonlinear dynamics and chaos. Chichester: Wiley.

Troger, H. \& Steindl, A. 1991 Nonlinear stability and bifurcation theory. Wien: Springer.

Weidner, C. \& Lechner, G. 1990 Klapper- und Rasselgeräusche in Fahrzeuggetrieben. Auto-tech. Z. 92, 320-326.

Yorke, J. \& Li, T.-Y. 1975 Period three implies chaos. Am. Math. Monthly 82, 985-992. 